\title{
Huge uterine STUMP (Smooth-muscle Tumor of Uncertain Malignant Potential) associated with extensive retroperitoneal lymphocystic malformations
}

Nour El-Etreby, Geylan Fadali, Khaled Atta Alexandria University, Egypt

Introduction: Uterine Fibromyomas are the most common benign tumors of the female genital system, with a reported incidence up to $40 \%$ in women during their reproductive years. But, not all uterine fibroids are completely benign. There is a rather obscure subgroup of these tumors that may have some grave characteristics such as the ability for recurrence or distant spread. This specific subgroup is called Atypical proliferative fibroid by some authors, other authors name it (STUMP) Smooth-muscle Tumor of Uncertain Malignant Potential. The classification of STUMP includes various combinations of the 3 main histologic determinants of smooth muscle tumors behavior which are the mitotic count, degree of cellular atypia and presence of coagulative necrosis.

Methods: We report a 49-years-old lady presenting with a huge solid pelviabdominal mass. After meticulous clinical and radiologic evaluation, the mass was proven to be of uterine origin, and associated with large and extensive lymphocystic formations related to the back of the uterus, broad ligament base as well as pelvic and para-aortic retroperitoneal spaces reaching up to the level of the renal vessels.

Results: We succeeded to excise the uterine mass and the large lymphocyst. The expert pathologic opinion was in favor of uterine (STUMP).

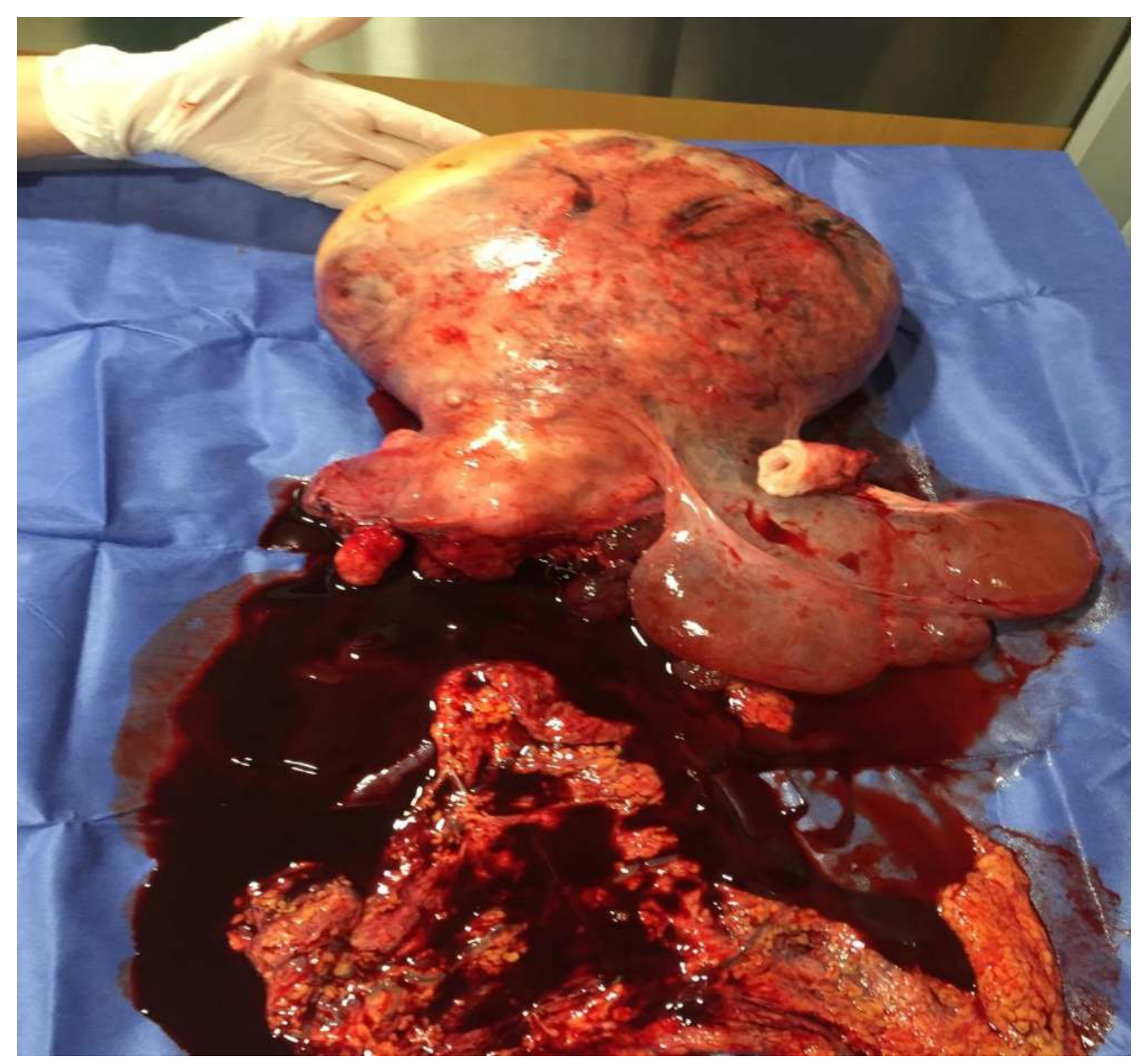

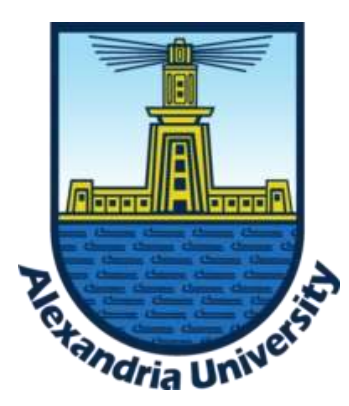

The patient is now nearly 9 months postoperative and her follow-up is completely free. The best treatment option in cases of STUMP is to perform a total hysterectomy; although myomectomy remains a viable option in cases with fertility desire, yet with unclear incidence of recurrence

Conclusions: To our knowledge, this is the first case in the literature to describe the association of a uterine STUMP with extensive retroperitoneal lymphocystic malformations. Our clinical judgement on the cause of these abnormal lymphocystic structures is the mass pressure-occlusion effects on the Lumbar Lymph Trunks. Uterine STUMP is a peculiar and rather obscure subgroup of uterine fibroids that requires more studies to enlighten its specific pathologic and clinical characteristics. A multidisciplinary approach to these tumors is crucial in achieving the best outcomes.
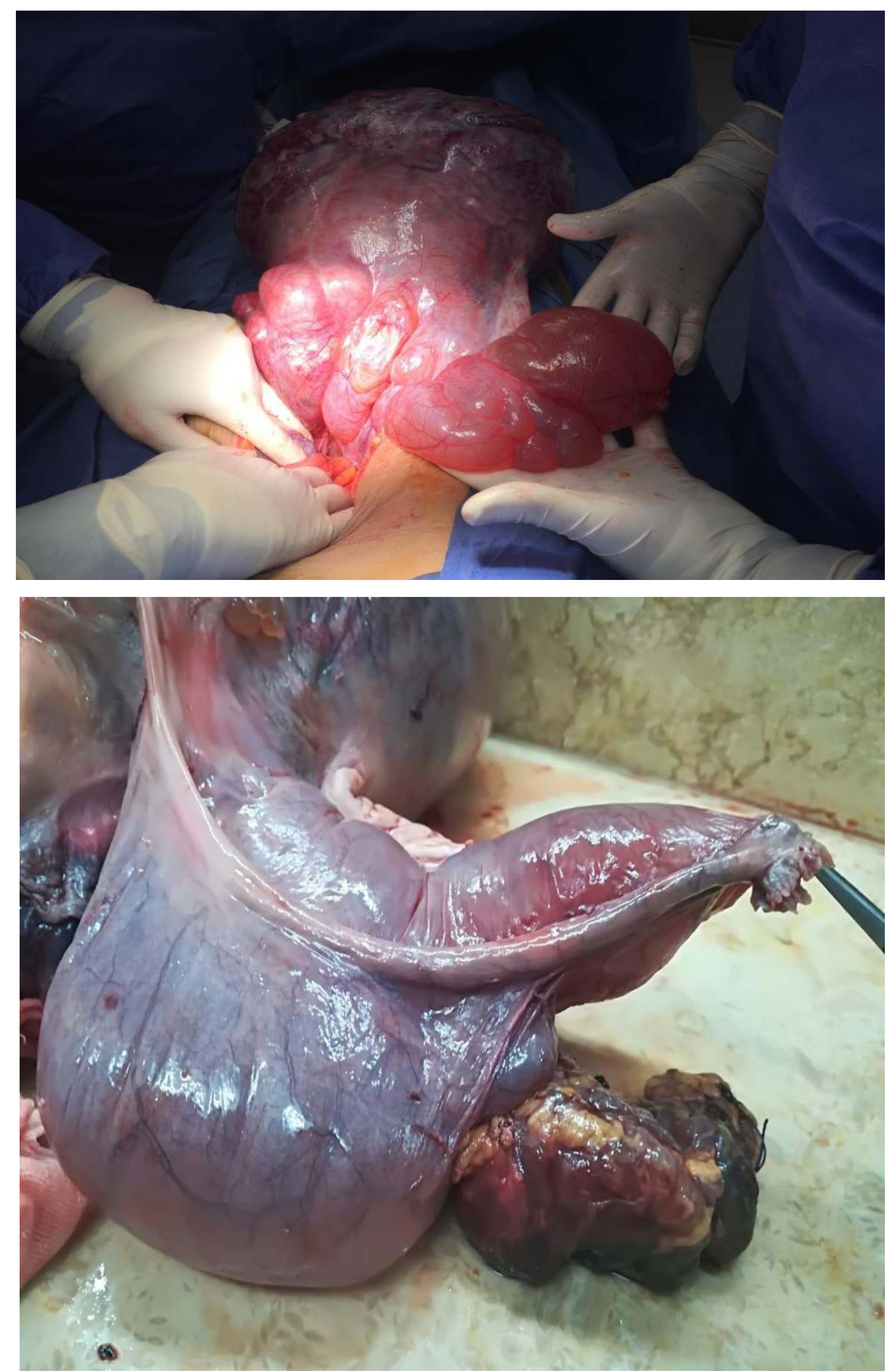